\title{
A Space-Time Multiscale Method for Parabolic Problems with Space- and Time-Dependent Coefficients
}

\author{
P. Ljung*, R. Maier* and A. Målqvist* \\ * Department of Mathematical Sciences \\ Chalmers University of Technology and University of Gothenburg \\ 41296 Gothenburg, Sweden
}

\begin{abstract}
In this presentation we study numerical solutions to a parabolic equation where the diffusion varies rapidly in both space and time. This type of equation, i.e. where the coefficient is highly varying, is commonly referred to as a multiscale equation, and it generally appears in the modelling of, for example, porous medium or composite materials. The parabolic equation arises in several real life applications, such as heat transfer and modelling of pressure in compressible flow. In particular, the time-dependency in the diffusion becomes of significance when considering a head conductor undertaking radioactive decay [3].

It is generally known that convergence of optimal order of the finite element method with piecewise linear polynomials relies on spatial $H^{2}$-regularity. Consequently, the optimal convergence rate can only be achieved once the mesh size is able to resolve the high variations in the diffusion, which quickly becomes computationally challenging. For this purpose, several so called multiscale methods have been developed to deal with these types of equations specifically. In this presentation, we propose a space-time multiscale method based on the framework of the localized orthogonal decomposition (LOD) method.

The LOD method was first introduced in [2] and have since then been analyzed for several different equations. In particular, in [1] the LOD method is analyzed for linear and semilinear multiscale parabolic equations, in which convergence of optimal order is proven. However, in this paper the diffusion coefficient is independent of time, and the corresponding LOD method fails to take rapid temporal variations into account. The main goal in this paper is to extend the LOD method into a generalized finite element method that deals with space-time multiscale equations.

The novel method is constructed by considering coarse test and trial spaces defined on the full spacetime domain. By utilizing a given interpolant, the fine-scale counterparts of these spaces may be defined, such that the full spaces are decomposed as a sum of its coarse and fine part. With the fine spaces defined, we can incorporate the fine behavior of the diffusion by solving corrector problems on the fine scale and include these in the coarse problem, such that the multiscale part of the diffusion is accounted for. Solving these corrector problems on the fine scale is however not computationally viable. To circumvent this complexity, we show that these correctors decay exponentially in both space and time, so that they can be computed on local patches, making the problem feasible.
\end{abstract}

\section{REFERENCES}

[1] A. Målqvist and A. Persson. Multiscale techniques for parabolic equations. Numerische Mathematik, 138(1):191-217, 2018.

[2] A. Målqvist and D. Peterseim. Localization of elliptic multiscale problems. Math. Comp., 83(290):2583-2603, 2014.

[3] S. Yousefi, D. Lesnic and Z. Barikbin. Satisfier function in ritz-galerkin method for the identification of a time-dependent diffusivity. Journal of Inverse and Ill-Posed Problems, 20, 12 2012. 